\title{
ERRATUM
}

\section{Coastal occupation and foraging during the last glacial maximum and early Holocene at Waterfall Bluff, eastern Pondoland, South Africa - Erratum}

Erich C. Fisher, Hayley C. Cawthra, Irene Esteban, Antonieta Jerardino, Frank H. Neumann, Annette Oertle, Justin Pargeter, Rosaria B. Saktura, Katherine Szabó, Stephan Winkler, Irit Zohar

DOI: https://doi.org/10.1017/qua.2020.26; Published online by Cambridge University Press: 14 May 2020

In the original publication of Fisher et al. (2020), a typesetting error occurred in Table 3 . The correct Table 3 is reproduced below. In addition, an error was introduced in the text referencing the samples described in Table 3. The correct text is written below:

For samples CN312, CN310, CN309, and CN308, further work on understanding site formation and sediment context at the microscopic level is required to make an informative interpretation of the $\mathrm{D}_{\mathrm{e}}$ distributions and to help choose an appropriate age model for final $\mathrm{D}_{\mathrm{e}}$ determination.

The publisher apologizes for these errors.

\section{Reference}

Fisher, E., Cawthra, H., Esteban, I., Jerardino, A., Neumann, F., Oertle, A., Pargeter, J. Coastal occupation and foraging during the last glacial maximum and early Holocene at Waterfall Bluff, eastern Pondoland, South Africa. Quaternary Research. First published online 14 May 2020. doi:10.1017/qua.2020.26.
Cite this article: Fisher, E. C. et al 2020. Coastal occupation and foraging during the last glacial maximum and early Holocene at Waterfall Bluff, eastern Pondoland, South Africa - Erratum. Quaternary Research 97, 216-217. https://doi.org/10.1017/qua.2020.55 


\begin{tabular}{|c|c|c|c|c|c|c|c|c|c|c|c|c|}
\hline \multirow{2}{*}{$\begin{array}{l}\text { Cat. } \\
\text { Number }\end{array}$} & \multirow{2}{*}{$\begin{array}{l}\text { Depth } \\
\text { (m) }\end{array}$} & \multirow[b]{2}{*}{ Sub-Agg } & \multirow{2}{*}{$\begin{array}{c}\text { Field water } \\
\text { content }(\%)^{*}\end{array}$} & \multicolumn{3}{|c|}{ Dose rates (Gy/ka) } & \multirow{2}{*}{$\begin{array}{c}\text { Total dose } \\
\text { rate }(\mathrm{Gy} / \mathrm{ka})\end{array}$} & \multirow{2}{*}{$\begin{array}{l}\text { Number } \\
\text { of grains }\end{array}$} & \multirow{2}{*}{$\begin{array}{c}\text { Age } \\
\text { Model }\end{array}$} & \multirow[b]{2}{*}{$\mathrm{D}_{\mathrm{e}}(\mathrm{Gy})$} & \multirow[b]{2}{*}{ OD $(\%)^{\#}$} & \multirow{2}{*}{$\begin{array}{l}\text { OSL age } \\
\text { (ka) }\end{array}$} \\
\hline & & & & Beta & Gamma & Cosmic & & & & & & \\
\hline 311 & 0.34 & SRCS Courtney & $22(22.5)$ & $0.397 \pm 0.024$ & $0.429 \pm 0.021$ & $0.052 \pm 0.004$ & $0.911 \pm 0.034$ & $123 / 1000$ & CAM & $12.2 \pm 0.4$ & $36.7 \pm 2.8$ & $13.3 \pm 0.7$ \\
\hline 310 & 0.45 & LBCS Kuka & $5(5.7)$ & $0.849 \pm 0.031$ & $0.578 \pm 0.026$ & $0.051 \pm 0.004$ & $1.511 \pm 0.041$ & $78 / 1000$ & $\begin{array}{l}\text { CAM } \\
\text { MAM }\end{array}$ & $\begin{array}{l}34.9 \pm 2.1 \\
19.0 \pm 1.8\end{array}$ & $45.9 \pm 4.7$ & $\begin{array}{l}23.1 \pm 1.6 \\
\mathbf{1 2 . 5} \pm \mathbf{1 . 2}\end{array}$ \\
\hline 309 & 0.57 & LBCS Otis & $10(10.8)$ & $0.877 \pm 0.037$ & $0.695 \pm 0.030$ & $0.050 \pm 0.004$ & $1.655 \pm 0.049$ & $317 / 3000$ & $\begin{array}{l}\text { CAM } \\
\text { MAM }\end{array}$ & $\begin{array}{l}66.1 \pm 1.9 \\
36.6 \pm 1.6\end{array}$ & $42.8 \pm 2.3$ & $\begin{array}{l}39.9 \pm 1.8 \\
\mathbf{2 2 . 1} \pm \mathbf{1 . 3}\end{array}$ \\
\hline 308 & 0.76 & LBCS Kate & $5(5.4)$ & $0.989 \pm 0.035$ & $0.740 \pm 0.033$ & $0.048 \pm 0.004$ & $1.809 \pm 0.049$ & $144 / 3000$ & $\begin{array}{l}\text { CAM } \\
\text { MAM }\end{array}$ & $\begin{array}{l}86.7 \pm 3.8 \\
50.3 \pm 3.4\end{array}$ & $42.8 \pm 3.6$ & $\begin{array}{l}47.9 \pm 2.7 \\
\mathbf{2 7 . 8} \pm \mathbf{2 . 1}\end{array}$ \\
\hline 312 & 0.82 & LBCS Colton & $5(4.1)$ & $1.058 \pm 0.038$ & $0.808 \pm 0.035$ & $0.047 \pm 0.004$ & $1.945 \pm 0.052$ & $66 / 2900$ & $\begin{array}{l}\text { CAM } \\
\text { MAM }\end{array}$ & $\begin{array}{r}100.9 \pm 7.4 \\
73.1 \pm 7.9\end{array}$ & $49.5 \pm 5.9$ & $\begin{array}{l}51.8 \pm 4.2 \\
\mathbf{3 7 . 6} \pm \mathbf{4 . 2}\end{array}$ \\
\hline
\end{tabular}

*Estimated water content over period of burial of sample. Current measured field water content value given in brackets.

${ }^{\#}$ Overdispersion (OD) values calculated using the CAM. 